\title{
Slope stability radar alarm threshold validation at Telfer gold mine
}

\author{
P Saunders GroundProbe Pty Ltd, Australia \\ S Nicoll Newcrest Mining Limited, Australia \\ C Christensen Newcrest Mining Limited, Australia
}

\begin{abstract}
The Telfer gold-copper mines in the Great Sandy Desert in the East Pilbara region of Western Australia lie 400 kilometres east-southeast of Port Hedland and approximately 1,300 kilometres by air or 1,900 kilometres by road north-east of the state's capital, Perth. Telfer comprises the Main Dome and West Dome open pits and the Telfer underground mine. GroundProbe Slope Stability Radars (SSR) are the primary monitoring tool at Telfer open pit mine for managing slope instability risk to personnel and equipment in a steep, brittle, hard rock environment.

This paper summarises the results of the back analysis of 15 collapses at Telfer gold mine and presents a discussion of the process developed by Geotechnical Support Services for application of SSR alarms in an open pit mining environment.
\end{abstract}

\section{Introduction}

The Alarm Threshold Validation and Back Analysis methodology described in this paper is a detailed strategy for application of SSR alarms for open pit mines. The methodology has been applied at Newcrest's Telfer mine and back analysis results are outlined, along with an explanation of the methods applied. The processes involved in the methodology provide a guideline for geotechnical engineers to assist with the development of a fully integrated site strategy for application of SSR alarms.

Applicable radar alarm thresholds are generated and collapse forecasting methods are tested for accuracy through back analysis of previous observed collapses. An ongoing, iterative approach to alarm threshold validation/justification has since been implemented on site, applying advanced velocity analysis techniques with repeated ongoing testing of alarm thresholds.

The methodology aims to optimise geotechnical risk management strategy and consequently contributes to minimising disruption to operations due to slope instability, whilst maintaining critical safety standards. It can be applied for all open cut mining methods including both hard and soft rock operations.

The methodology adopts a strategic approach to:

- Justify existing alarm thresholds by testing the effectiveness of thresholds applied for previous collapses.

- Generate and test new alarm thresholds applying the full suite of SSR-Viewer 8 (2013) stackable alarms.

- Integrate SSR-Viewer 8 (2013) stackable alarms with the site's geotechnical/radar Trigger Action Response Plan (TARP).

- Develop a collapse database to store historic collapse data and back analysis results.

The processes involved serve to determine appropriate alarm thresholds that can be applied to all open pit mining applications, without the requirement for a thorough understanding of failure mechanisms or rock 
mass characteristics. Defining critical velocity ranges and observing and documenting typical deformation trends that occur prior to collapse is also important. Selecting an appropriate time to exclude personnel or equipment prior to failure can be difficult and is generally based on a conservative judgement by site engineers with limited analysis to justify the parameters adopted. The aim then is to optimise this process and minimise costly delays while maintaining safe working conditions.

\section{Economic and safety impact}

Generally speaking, it may be seen as acceptable to apply a long exclusion period prior to collapse. Most operations would be satisfied if an alarm triggers a few days prior to collapse. However, early exclusion from valuable ore reserves and long production delays can be costly. Calculating this cost is relatively simple for most operations but is often neglected.

Analysis to develop an improved understanding and effectively manage the risk of continuing to operate in an area apparently at risk of collapse can be an extremely valuable exercise for a mining operation. Repeated exclusion or isolation from high value mining areas is not often questioned when the risk to personnel or equipment seems apparently too difficult to quantify. Where the risk of a significantly large collapse is identified, strong risk management controls such as isolation are often applied based on assumptions that an observed deformation rate is significant and presents a real risk, but in many cases the risk may not have been rigorously evaluated. Collapse forecasting methods are also not often applied in an operational environment due to lack of confidence, site experience, production pressures and time constraints.

Engineering due diligence is required when choosing critical cumulative deformation or velocity thresholds for alarms, as these are often considered the front-line-of-defence in high risk, high exposure mining areas. Without a carefully considered strategy for application of alarms for monitoring instruments such as radar, some operations may be left exposed, without appropriate documentation, processes or adequately trained site personnel. Inadequate training and/or documentation relating to alarm threshold justification should be recognised as a significant potential business risk for any operation wishing to reflect what should be seen as industry best practice. Determining appropriate alarm thresholds is a key component of a site's geotechnical risk management strategy with regard to slope stability monitoring with radar.

Effective real-time application of collapse forecasting techniques using velocity analysis software tools available in SSR-Viewer 8 (2013) can assist to not only manage the risk associated with wall collapse, but also minimise disruption to mining operations caused by overly conservative geotechnical exclusion times.

\section{Trigger action response plans}

GroundProbe's SSR-Viewer 8 (2013) software allows application of six different types of alarms which can be applied with thresholds designed to trigger in sequence leading up to collapse. These alarms are referred to as stackable alarms. Hierarchical alarms are set for each different alarm type with different thresholds intended to trigger in sequence. These alarms can be aligned with a site's geotechnical TARP; the triggering of sequential alarms leads to an associated site response which is described in the TARP document. A TARP is a policy of planned responses to trigger events. The purpose of a TARP is to define a range of trigger levels and the associated response protocols to be initiated in the event of a trigger level being exceeded. Figure 1 shows diagrammatically how the SSR-Viewer 8 (2013) alarms can be aligned with a site TARP. 


\section{- Deformation}

- Velocity

- Velocity Ratio

\section{- Inverse Velocity}

\section{- Coherence}

\section{- Tracking}

TARP Trigger 1 - Green - Normal

TARP Trigger 2 - Yellow - Caution

TARP Trigger 3 - Orange - Linear deformation

TARP Trigger 4 - Progressive deformation

Figure 1 Alignment of stackable alarms with TARP trigger levels

\section{$4 \quad$ Alarm application methodology}

Deformation and velocity alarms are generally the most frequently applied and easily understood alarm types for slope monitoring. Once an increased level of risk is identified (a linear deformation trend) the other SSR-Viewer 8 (2013) stackable alarms can be applied. Thresholds for preliminary alarms, when set with a broad area alarming strategy (minimal alarm masking), can be determined from back analysis. It is important to remember that this type of empirical approach by itself may be unreliable due to the small sample size and variability in failure mechanisms. From the author's experience, a broad area alarm strategy seems to be that which is most commonly applied in the industry; where minimal alarm masking is applied and no prior significant deformation trends have been observed. Alarm thresholds are often arbitrary or based loosely on site experience.

When applying a targeted approach, areas showing signs of deformation are isolated using masking and alarms are set for that area. Tight alarm masks are applied to prevent unwanted alarms being triggered by mining interaction or atmospheric events occurring outside the area of concern.

When a linear deformation trend is identified, the velocity is noted and a new velocity (or velocity ratio) alarm can be set. The aim of this alarm is to alert the user of the onset of a progressive deformation trend. As the slope begins to yield and progressive deformation is observed (accelerating slope movement), the associated risk increases and so does the assigned TARP Trigger level. At this stage, forecasting methods can be applied and inverse velocity alarms can be set. As the onset of failure occurs and new fractures begin to form, final stage alarms, coherence and tracking, can be set to alert the engineer that the surface of the slope is beginning to deform or fracture significantly between scans or the velocity is approaching the limits of the radar's ability to track the deformation effectively ( $>7.85 \mathrm{~mm} / \mathrm{scan}$ ). 


\section{$5 \quad$ Telfer analysis results}

Testing/validation of radar alarm thresholds is not common industry practice, yet the consequence of poorly considered alarm thresholds is significant in terms of safety risk and potential for significant production losses. Back analysis of historic collapses at Telfer gold mine was conducted to test the appropriateness of alarm thresholds applied on site. Alarm thresholds applied at the time of collapse were tested and the notification period prior to collapse documented. Test thresholds were applied for the full suite of SSR-Viewer 8 (2013) alarms and the sequence in which the alarms would have triggered prior to collapse was also recorded. A detailed velocity analysis to investigate typical slope velocities and deformation trends leading up to collapse was conducted and the effect of different velocity calculation periods was considered. The accuracy of collapse forecasting was also assessed using different velocity calculation periods. Table 1 lists the collapses that were analysed for alarm threshold validation.

Table 1 Telfer collapse records for analysis

\begin{tabular}{llllll}
\hline Rockfall report & Time of collapse & Lithology & Failure size & Failure mechanism \\
\hline TRFR-MD_S4_2015-11-06_1 & $6 / 11 / 201523: 40$ & OSM & $15,000 \mathrm{t}$ & Combination \\
\hline TRFR-MD_S4_2015-03-12_1 & $12 / 03 / 201510: 57$ & OSM & $2,500 \mathrm{t}$ & Combination \\
\hline TRFR-MD_S4_2015-01-09_1 & $9 / 01 / 20152: 28$ & OSM & $7,300 \mathrm{t}$ & Unravelling \\
\hline TRFR-MD_S4_2015-01-07_1 & $7 / 01 / 201521: 42$ & OSM & $35,000 \mathrm{t}$ & Rockmass \\
\hline TRFR-MD_S4_2015-09-07_1 & $7 / 09 / 2015$ 19:26 & OSM & $250 \mathrm{t}$ & Unravelling \\
\hline TRFR-MD_S4_2015-09-11_1 & $11 / 09 / 2015$ 21:26 & MU & $250 \mathrm{t}$ & Rockmass \\
\hline TRFR-MD_S4_2015-07-14_1 & $14 / 07 / 201510: 51$ & OSM & $600 \mathrm{t}$ & Rockmass \\
\hline TRFR-MD_S4_2015-07-15_1 & $15 / 07 / 2015$ 9:07 & OSM & $600 \mathrm{t}$ & Unravelling \\
\hline TRFR-MD_S4_2015-09-20_1 & $20 / 09 / 2015$ 9:29 & MU & $1,250 \mathrm{t}$ & Rockmass \\
\hline TRFR-MD_S4_2015-02-10_1 & $10 / 02 / 2015$ 1:15 & OSM & $3,000 \mathrm{t}$ & Unravelling \\
\hline TRFR-MD_S4_2015-02-24_1 & $24 / 02 / 2015$ 9:02 & OSM & $200 \mathrm{t}$ & Unravelling \\
\hline TRFR-MD_S4_2015-03-04_1 & $4 / 03 / 20155: 01$ & OSM & $4,000 \mathrm{t}$ & Rockmass \\
\hline TRFR-MD_S4_2015-04-30_1 & $30 / 04 / 2015$ 5:20 & OSM & $200 \mathrm{t}$ & Unravelling \\
\hline TRFR-MD_S4_2015-05-05_1 & $5 / 05 / 20154: 58$ & OSM & $180 \mathrm{t}$ & Unravelling \\
\hline
\end{tabular}

Velocities measured prior to collapse were analysed considering rates in both $\mathrm{mm} / \mathrm{hr}$ and $\mathrm{mm} /$ day and applying 60, 180, 720 and 1,440 minute velocity calculation periods (VCP).

Conclusions drawn from the results of the study were as follows:

- A velocity threshold of $1.5 \mathrm{~mm} / \mathrm{hr}$ and equivalent inverse velocity of $0.66 \mathrm{hr} / \mathrm{mm}$ proved to be appropriate with an average notification period of 6.96 hours and an observed minimum notification period of 1 hour, 24 minutes.

- Deformation alarm thresholds of $4 \mathrm{~mm} / 4 \mathrm{hrs}$ and $6 \mathrm{~mm} / 4 \mathrm{hrs}$ were tested and proved appropriate with alarms triggering, on average, $>5$ hours prior to failure.

- Inverse velocity alarms of $0.2 \mathrm{hr} / \mathrm{mm}$ or less, coherence and tracking alarms were determined to be inappropriate in terms of providing adequate notification periods for Telfer providing an average notification period of $<1$ hour prior to failure. 
Using SSR Viewer, the alarms in Table 2 were tested for each collapse. The average, maximum and minimum notification period was recorded for each alarm test threshold and type.

Table 2 Telfer alarm back analysis results

\begin{tabular}{|c|c|c|c|c|}
\hline Alarm type & Generic threshold & Average (hrs) & Maximum & Minimum \\
\hline \multirow{2}{*}{ Deformation } & $4 \mathrm{~mm} / 4 \mathrm{hrs}$ & 6.33 & 10 hours 45 minutes & 1 hour 24 minutes \\
\hline & $8 \mathrm{~mm} / 4 \mathrm{hrs}$ & 3.22 & 9 hours 52 minutes & 0 minutes \\
\hline \multirow{2}{*}{ Velocity } & $3 \mathrm{~mm} / \mathrm{hr}$ & 2.3 & 12 hours 15 minutes & 0 minutes \\
\hline & $2 \mathrm{~mm} / \mathrm{hr}$ & 2.71 & 8 hours 34 minutes & 0 minutes \\
\hline \multirow{2}{*}{ Velocity ratio } & 2 & 2.03 & 7 hours 27 minutes & 13 minutes \\
\hline & 2 & 2.42 & 7 hours 27 minutes & 0 minutes \\
\hline \multirow{4}{*}{$\begin{array}{l}\text { Inverse } \\
\text { velocity }\end{array}$} & $0.66 \mathrm{hr} / \mathrm{mm}$ & 6.96 & 20 hours 39 minutes & 1 hour 24 minutes \\
\hline & $0.4 \mathrm{hr} / \mathrm{mm}$ & 2.77 & 7 hours 44 minutes & 0 minutes \\
\hline & $0.3 \mathrm{hr} / \mathrm{mm}$ & 1.66 & 3 hours 53 minutes & 0 minutes \\
\hline & $0.2 \mathrm{hr} / \mathrm{mm}$ & 0.82 & 2 hours 3 minutes & 0 minutes \\
\hline Coherence & 0.9 & 0.33 & 1 hour 14 minutes & 0 minutes \\
\hline Tracking & $6.85 \mathrm{~mm} / \mathrm{scan}$ & 0.21 & 39 minutes & 0 minutes \\
\hline
\end{tabular}

Data from the collapse with rockfall report reference TRFR-MD_S4_2015-07-15_1 (refer to Table 1) was excluded from analysis as this was considered an extension of the collapse TRFR-MD_S4_2015-07-14_1. Data from TRFR-MD_S4_2015-04-30_1 and TRFR-MD_S4_2015-05-05_1 were also excluded from the analysis as these failures were considered extensions of the collapse TRFR-MD_S4_2015-03-04_1.

Considering the average notification periods of the thresholds tested and presented in Table 2 , a $1.5 \mathrm{~mm} / \mathrm{hr}$ velocity alarm or $0.66 \mathrm{hr} / \mathrm{mm}$ inverse velocity alarm provided the longest notification period prior to collapse at 6.96 hours and a minimum notification period of 1 hour, 24 minutes. Deformation alarms of $4 \mathrm{~mm} / 4 \mathrm{hrs}$ and $6 \mathrm{~mm} / 4 \mathrm{hrs}$ also provided appropriate notification periods of 6.33 and 5.74 hours respectively. The shortest notification period, for each of these alarm types, was at least one hour prior to failure. Coherence alarms tracking alarms and inverse velocity alarms of less than $0.2 \mathrm{hr} / \mathrm{mm}$, were considered inappropriate based on this analysis providing notification periods of $<1$ hour prior to failure.

Histograms in Figures 2 and 3 show the number of collapses that would have triggered particular alarm thresholds for given notification period ranges. A velocity alarm of $1.5 \mathrm{~mm} / \mathrm{hr}$ was tested by applying an equivalent inverse velocity threshold of $0.66 \mathrm{hr} / \mathrm{mm}$. Velocity ratio alarms were tested by choosing a reference period at the first noticeable occurrence of a linear deformation trend and setting an alarm to trigger at a multiple of that magnitude.

The red bars represent the number of alarms providing $<1$ hour notification period prior to collapse. This was considered an inadequate time to allow communication of risk and to allow for safe evacuation. The orange bars correspond to alarms that triggered 1-3 hours prior to failure. This was considered a reasonable notification period, but not ideal. The green bars illustrate alarms that triggered $>3$ hours prior to failure. This was considered a minimum acceptable notification period prior to collapse for Telfer. 


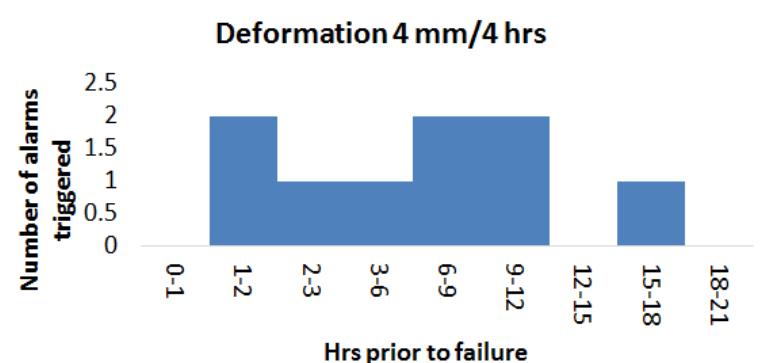

Velocity $2 \mathrm{~mm} / \mathrm{hr}$ over $60 \mathrm{mins}$

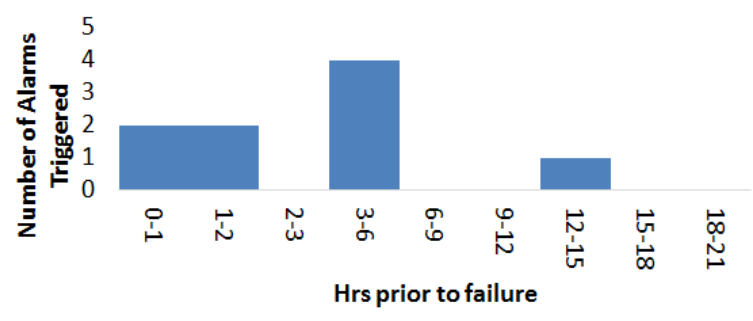

Inverse velocity $0.66 \mathrm{hr} / \mathrm{mm}$

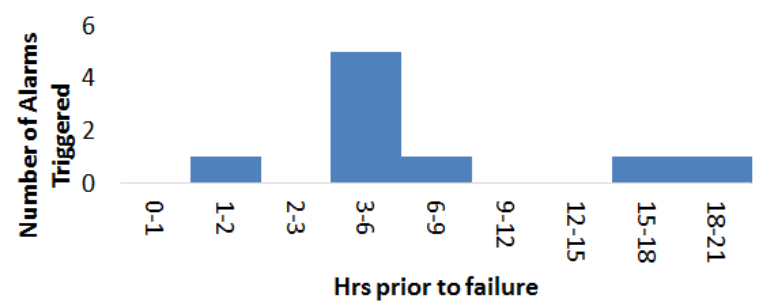

Inverse velocity $0.3 \mathrm{hr} / \mathrm{mm}$

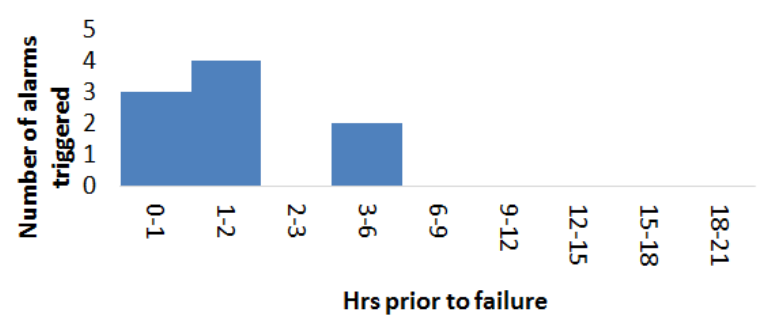

Figure 2 Notification periods for test alarm triggers

Velocity ratio 2 over $60 \mathrm{~min}$

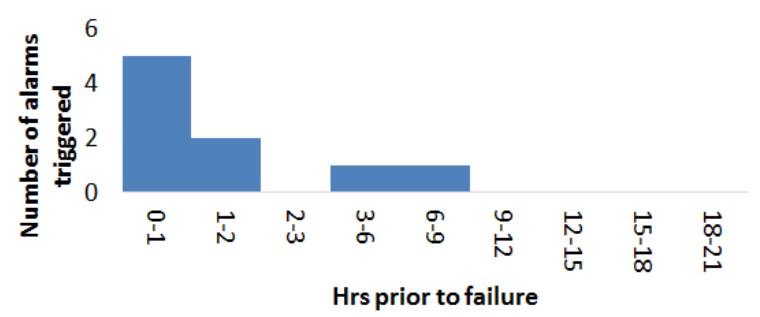

Coherance 0.9

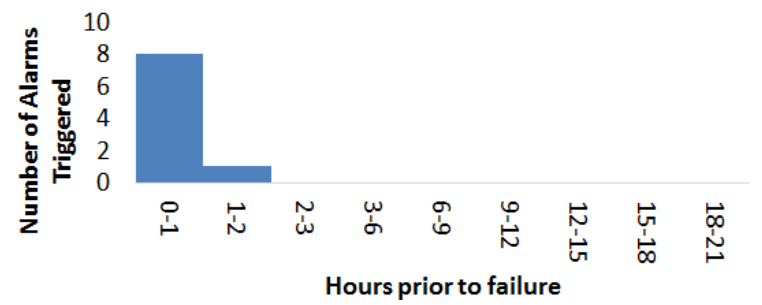

Figure 3 Notification periods for test alarm triggers

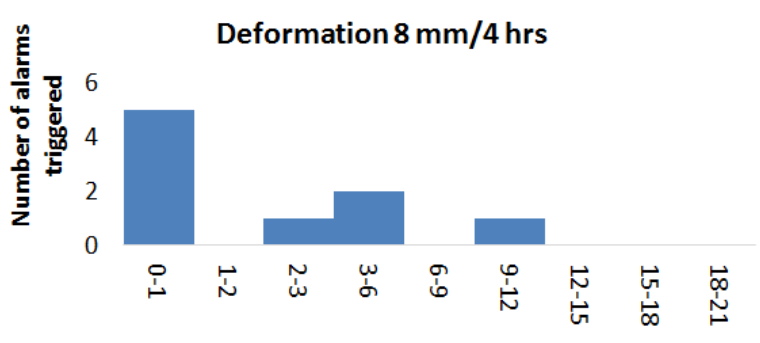

Hrs prior to failure

Velocity $3 \mathrm{~mm} / \mathrm{hr}$ over $60 \mathrm{mins}$

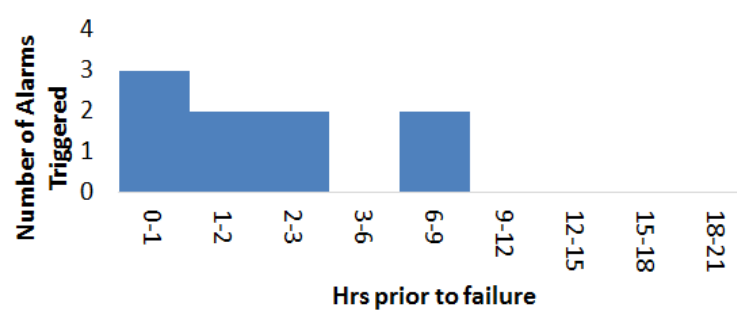

Inverse velocity $0.4 \mathrm{hr} / \mathrm{mm}$

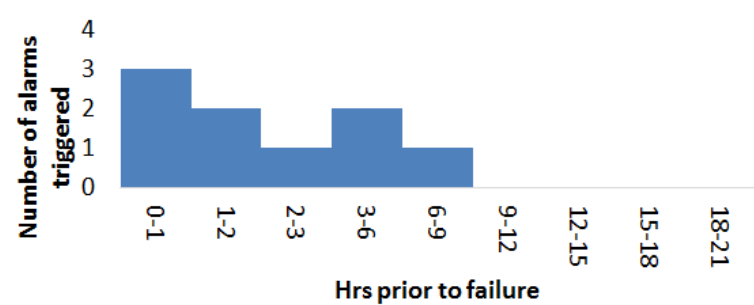

Inverse Velocity $0.2 \mathrm{hr} / \mathrm{mm}$

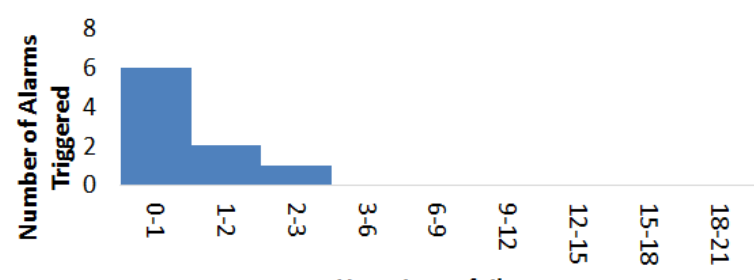

Hrs prior to failure

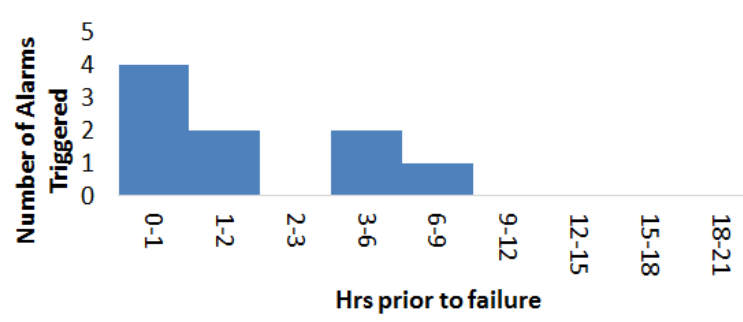

Tracking $6.85 \mathrm{~mm} / \mathrm{scan}$

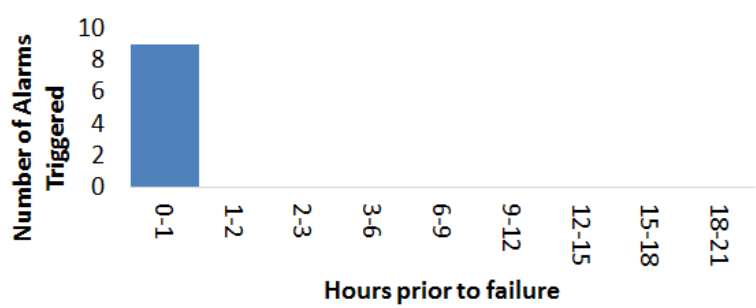




\subsection{Forecasting collapse time}

The accuracy of the inverse velocity method to forecast failures at Telfer was investigated by applying various VCP. The results concluded that:

- Application of a 60 minute VCP resulted in a collapse forecast between two hours prior and three hours post-failure.

- A 180 minute VCP resulted in a collapse forecast between one hour prior and four hours post-failure.

- 720-1,440 minute VCP resulted in a collapse forecast time up to 14 hours post-failure.

Although larger calculation periods produced less accurate failure forecast times, results showed that large time windows were useful for detection of progressive trends as early as 36 hours before failure.

Figure 4 displays the inverse velocity values measured at, or very close to, the time of collapse applying different velocity calculation periods. The majority of collapses occurred with inverse velocity reaching values below $1.5 \mathrm{~h} / \mathrm{mm}$. From this analysis it was clear that applying a longer VCP resulted in higher measured inverse velocity values and applying a shorter VCP resulted in a lower calculated inverse velocity at time of collapse. From this it could be concluded that, for forecasting purposes, applying shorter velocity calculation periods resulted in a more accurate collapse forecast.
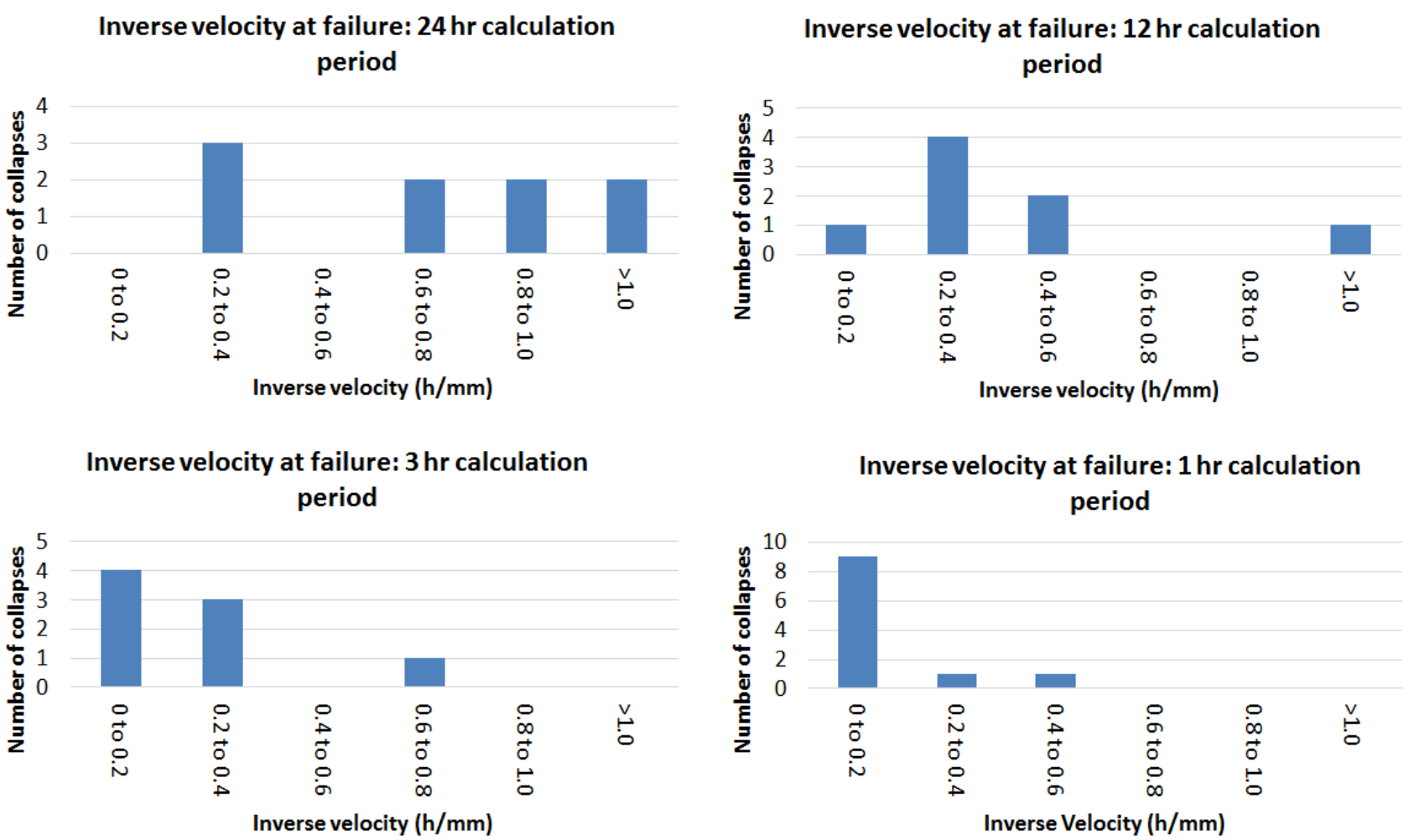

Figure 4 Inverse velocity at time of collapse with variable VCP 


\subsection{Collapse forecast error}

For each collapse, a forecast was made based on the available data at some convenient point in time prior to failure. This time was chosen to be a number of hours prior to the collapse, at which point there would be adequate time to evacuate personnel and machinery from the area of concern.

The error between the actual failure time and the forecasted failure time was measured for each collapse. The histograms in Figure 5 display the forecast error for different velocity calculation periods. The green bars correspond to events that forecast within one hour of the actual failure time. The orange bars correspond to events where the failure prediction was one to two hours before or after the actual failure event. The red bars correspond to cases where the failure prediction was greater than two hours after the actual failure time. It can be concluded from this that a shorter velocity calculation period will provide a more accurate forecast prediction.

Forecast error using inverse velocity $24 \mathrm{hr}$

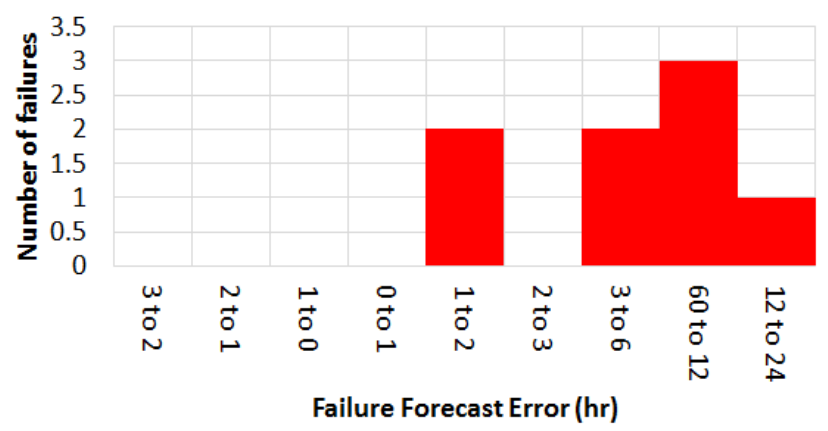

Forecasting error using inverse velocity $3 \mathrm{hr}$

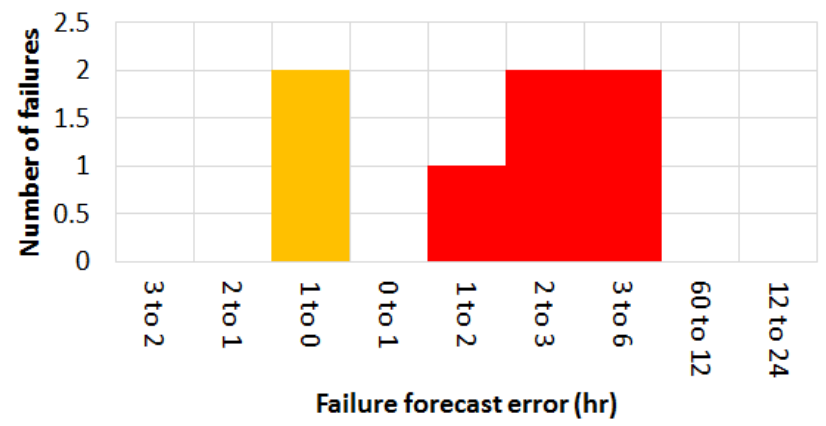

Forecast error using inverse velocity $12 \mathrm{hr}$

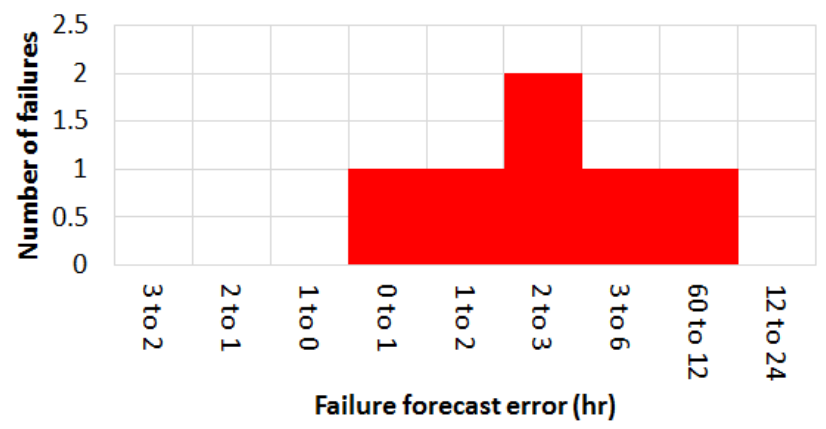

Forecast error using inverse velocity $1 \mathrm{hr}$

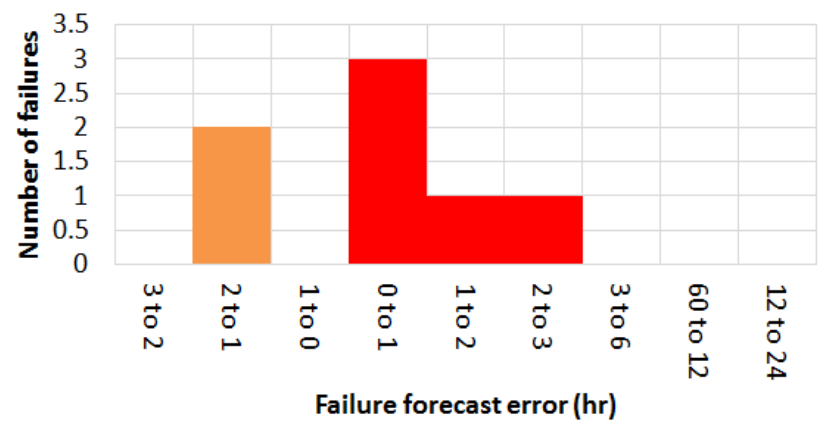

Figure 5 Forecast error (pre and post-collapse) applying variable VCP

\subsection{Velocity analysis}

The velocity prior to collapse was examined for each of the failures listed in Table 1 . This was done in order to identify and analyse deformation trends prior to failure. The analysis was done using both $\mathrm{mm} / \mathrm{hour}$ and $\mathrm{mm} /$ day rates. Common trends can be seen when comparing the graphs in Figure 6 . A rapid increase in velocity was typically observed two hours prior to failure when considering velocity in $\mathrm{mm} / \mathrm{hr}$, with peak velocity rates ranging from 2.23 to $21.9 \mathrm{~mm} / \mathrm{hr}$. A common trend appeared between collapses when considering velocity in $\mathrm{mm} /$ day where velocity began to rapidly increase one day prior to collapse, with peak velocity ranging from 11.0 to $58.0 \mathrm{~mm} /$ day. 

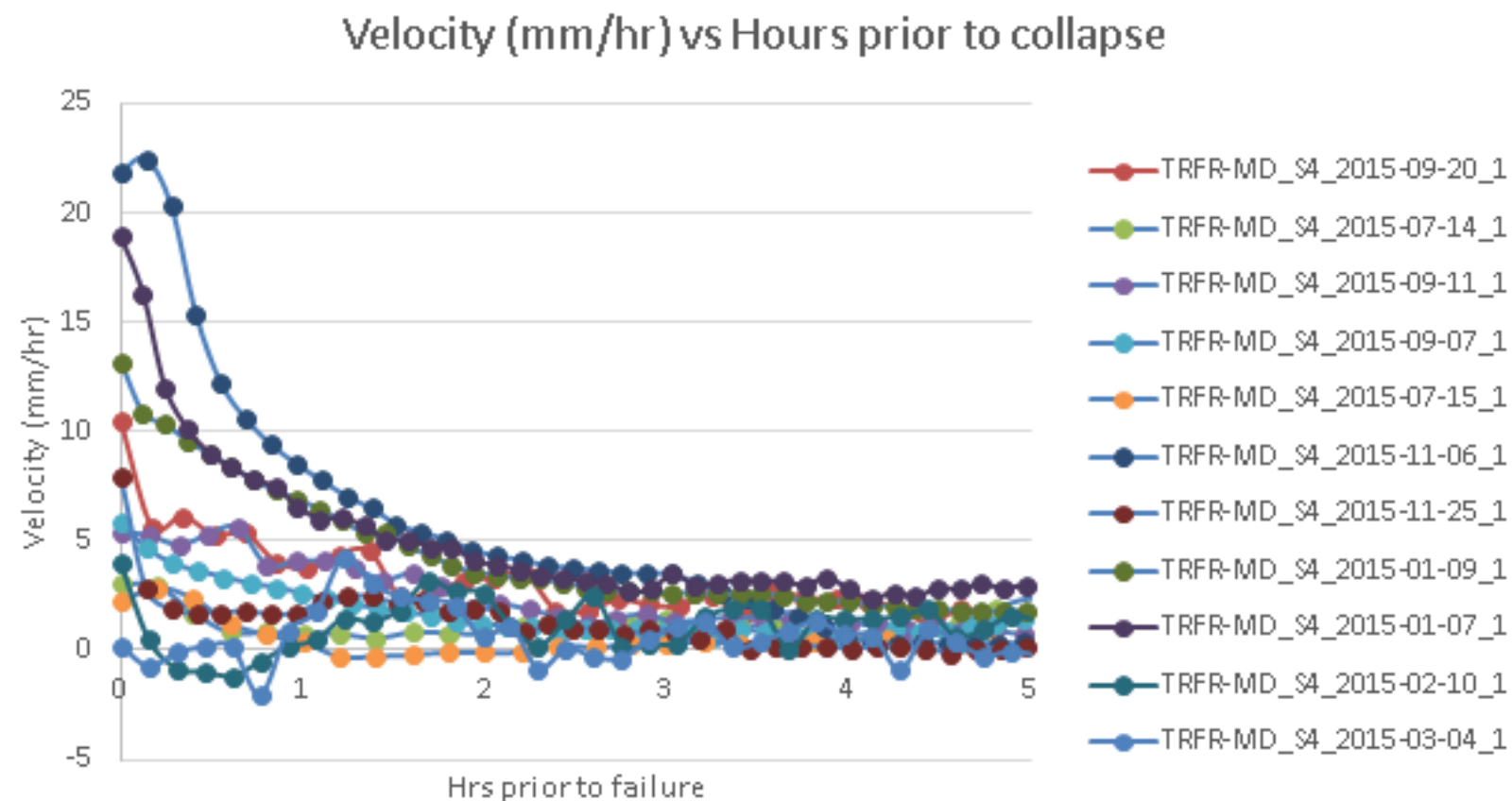

Hrs prior to failure

Velocity $(\mathrm{mm} /$ day) vs Days prior to collapse
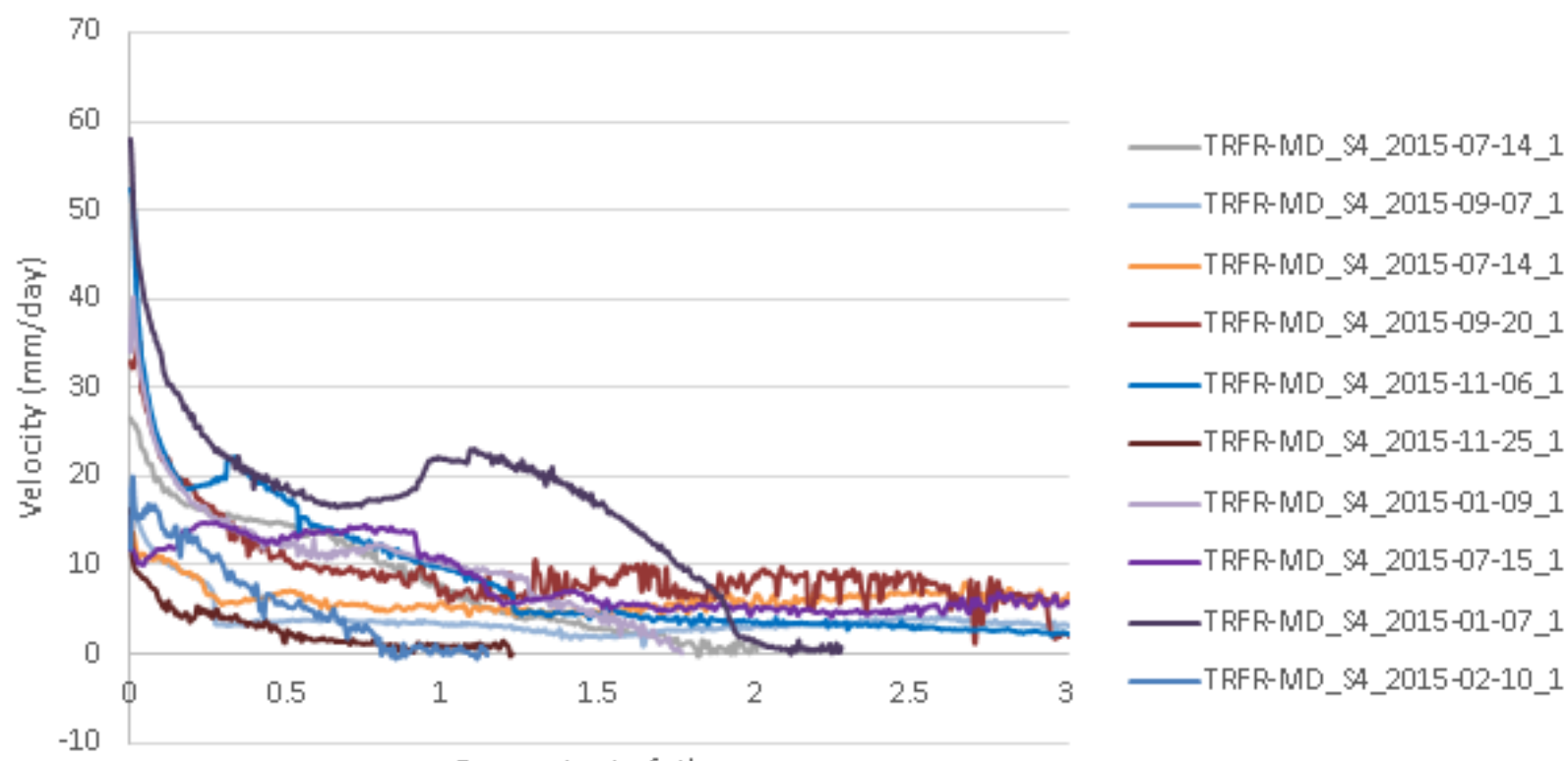

Days prior to failure

Figure 6 Velocity graphs leading up to collapse in $\mathrm{mm} / \mathrm{hr}$ compared to $\mathrm{mm} /$ day

Analysis of the velocity trend of TRFR-MD_S4_2015-01-07_1 prior to collapse shows two noteworthy periods of progressive deformation which can be seen in Figure 7. The first process occurred two days prior to failure, reaching a maximum velocity of $20 \mathrm{~mm} /$ day. This deformation process was believed to be induced by excavation of broken material near the toe of the wall. The wall movement slowed when excavator activity stopped. The second process was twelve hours prior to failure, when the excavator continued activity below the wall, which caused wall movement to accelerate to approximately $59 \mathrm{~mm} /$ day to eventual failure. 


\section{Velocity (mm/day) vs Days prior to collapse}

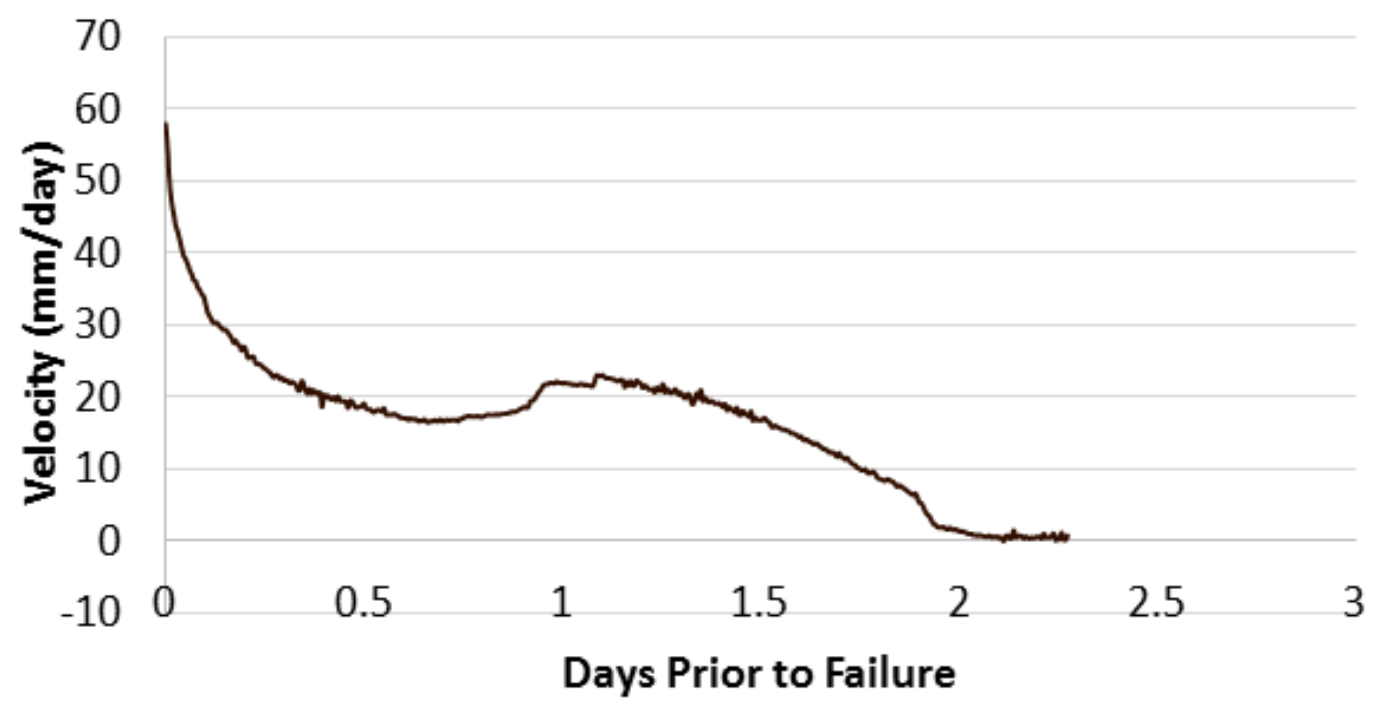

Figure 7 Velocity trend of TRFR-MD_S4_2015-01-07_1 prior to collapse

Comparing velocity over various time periods ( $\mathrm{mm} / \mathrm{hr}$ and $\mathrm{mm} /$ day) was found to be a useful analysis technique. By comparing the two, the larger time window in $\mathrm{mm} /$ day was found to be useful for detecting ongoing deformation processes that may be occurring over a longer period prior to failure. From this investigation it was noted that detection of progressive deformation processes at Telfer, suggesting an area is trending towards collapse, can be as early as 36 hours prior to failure.

\section{Velocity calculation period}

Velocity is calculated in the SSR-Viewer 8 (2013) software simply by dividing the measured cumulative deformation by the specified time window. Hence the gradient of the secant to the curve is measured rather than the instantaneous velocity, which of course would normally be calculated by the differential of the curve equation. This simplified method for velocity calculation, when monitoring slopes with radar, leads to different results when using different length time windows. This must also be taken into consideration particularly when applying the inverse velocity method to forecast collapse times (Cabrejo-Liévano 2013). Using a longer calculation period will result in a less accurate forecast collapse time. A balance must be found between using a short calculation period with less usable 'noisy' data and smooth data that shows a clear trend when using a longer calculation period.

It was found from the Telfer study that velocity increased rapidly during the two hours prior to failure when applying a 60 minute VCP, with peak velocities reaching values of 2.23 to $21.9 \mathrm{~mm} / \mathrm{hr}$. When applying a 1,440 minute $V C P$, it was noted that the velocity in all cases showed a rapid increase one day prior to slope failure, allowing early identification of a significant change in deformation trend, with peak velocity rates ranging from 11.0 to $58.0 \mathrm{~mm} /$ day.

Collapses at Telfer can generally be described as rapid, brittle failures and, hence, relatively high velocity thresholds will not generally provide an adequate notification period prior to collapse. In a soft rock environment, high peak velocities are often observed prior to functional collapse. Applying a velocity alarm threshold of $1 \mathrm{~mm} / \mathrm{hr}$ with a short 60 minute VCP resulted in frequent unwanted alarms due to data 'noise'. A threshold of $2 \mathrm{~mm} / \mathrm{hr}$ resulted in an insufficient notification period when tested on previous collapses. A $1.5 \mathrm{~mm} / \mathrm{hr}$ velocity was found to be appropriate in all cases tested. It is likely that this value could be further refined with repeated ongoing back analysis. 
Although it was found that using a longer velocity calculation period produced less accurate failure forecast times, results showed that large time windows were useful for early detection of significant changes in deformation trends, allowing early notification of collapse trends.

\section{Deformation alarm thresholds}

Although deformation alarms are rarely applied on site at Telfer, the results of the back analyses conducted suggest that their application may provide an additional layer of control when applied in conjunction with velocity and other stackable alarms. A deformation magnitude threshold of $4 \mathrm{~mm}$ triggered in all scenarios with adequate notification, although a very small rock fall analysed was observed to trigger this alarm only 49 minutes prior to collapse. Hence a deformation alarm alone may not be adequate for very small falls of ground. As this collapse was approximately the same size as one pixel, the contiguous pixels parameter would need to have been set to one. In all other test cases though, a $4 \mathrm{~mm} / 4 \mathrm{hrs}$ deformation threshold would have provided an appropriate notification period prior to collapse.

A $1 \mathrm{~mm} / \mathrm{hr}$ velocity alarm had been applied on site previously with limited success due to frequent unwanted alarms caused by noise in the data. A $4 \mathrm{~mm} / 4 \mathrm{hr}$ deformation threshold alarm could be applied as a viable alternative, representing the same deformation rate but being less susceptible to triggering of unwanted alarms.

In all cases, and with regard to all alarms, some degree of engineering judgement must be applied. Careful data analysis, looking for changes in the deformation trend prior to collapse, is critical. The significance of cumulative deformation measured prior to collapse is dependent on the modulus of the rock mass on a large scale, which may be highly variable due to local structural features affecting the stiffness of the overall system.

\section{$8 \quad$ New mining projects}

For new mining areas or projects where there is no history of collapse or benchmarking data, the question arises: What alarm thresholds should be set? The influence of variability in failure mechanisms, internal shear strength and rock stiffness may suggest that back analysis of past collapses in a slope may be unreliable for determining appropriate alarm thresholds given the small sample size. Back analysis can arguably be used to determine preliminary velocity alarm thresholds, but knowing that no two collapses will ever be the same suggests that a more diligent, multi-layered approach is required. Applying a quantitative velocity or deformation alarm threshold should be only a small part of a more detailed strategic approach to slope monitoring and managing the risk of collapse.

Firstly, it is important to understand the purpose of an alarm. Typically, an alarm is set to alert the user to a change in deformation trend. It is common practice for mining operations to exclude personnel and equipment from areas where progressive deformation is observed. Once a clear progressive trend is seen in an area, regular, repeated collapse forecasting can be applied using the inverse velocity method. With a well-defined process for collapse forecasting (regular forecasts with updated data, continuous real-time human monitoring, i.e. an engineer continuously monitoring the live data) it may be possible to reduce the risk to an acceptable level in some circumstances to permit continued mining in areas where the slope is progressively deforming. This concept has potential to add significant value to some operations, particularly 'soft systems' where slopes may accelerate over extended periods (weeks or months) prior to functional collapse.

Without any history of collapse or benchmarking data, monitoring data in real time, measuring velocity and setting alarms to trigger if an area begins to deform at a velocity higher than the current observed rate is the only reliable way to determine appropriate preliminary alarm thresholds. 


\section{Conclusion}

Back analysis of previous collapses can provide an operation with defensible alarm thresholds for a range of alarm types. This back analysis process can also be used to assess the effectiveness of the inverse velocity method in predicting failure times.

This paper describes the methodology developed by the primary author as applied at Telfer gold mine and the results of the back analysis work conducted. The analysis showed that a longer velocity calculation period can be applied for early warning of the onset of collapse. The complete methodology for application of alarms and back analysis describes the application of the full suite of SSR stackable alarms. Targeted and broad area alarm masking approaches are described as part of the methodology, along with the concept of incorporation and alignment of the SSR stackable alarms with a site TARP.

The results of this study effectively represent an empirical model for alarm threshold validation at Telfer gold mine based on back analysis of failures ranging in size from 250 to 35,000 tonnes. The purpose of the model was to validate alarm settings for a range of failure sizes and failure mechanisms and to assess, in particular, the applicability of the inverse velocity method for predicting time of collapse at Telfer gold mine.

Back analysing failures in the Telfer Main Dome open pit has provided a better understanding of the SSR monitoring system for site engineers and provided documentation to justify the application of specific alarm types and thresholds. A velocity alarm threshold of $1.5 \mathrm{~mm} / \mathrm{hr}$ was shown to provide the most appropriate notification period most often, triggering on average 6.96 hours prior to failure. Deformation alarms of $4 \mathrm{~mm} / 4 \mathrm{hrs}$ and $6 \mathrm{~mm} / 4 \mathrm{hrs}$ were also deemed appropriate with alarms triggering more than five hours prior to failure.

The accuracy of the inverse velocity method to forecast failures was investigated over various velocity calculation periods. The inverse velocity value measured at failure was investigated and it was shown that shorter velocity calculation periods produced more accurate failure forecasts. The 1,440 minute velocity calculation period forecast error ranged from 0 to 14 hours post failure in comparison to the 60 minute calculation period forecast error ranging from two hours prior to three hours post failure.

The velocity prior to failure was analysed over 1 and 24 hour time windows. When observing velocity in the days leading to failure (measured in $\mathrm{mm} /$ day), significant trends were identified as early as 36 hours prior to collapse in all cases. In comparison, when observing the velocity in hours leading up to failure in $\mathrm{mm} / \mathrm{hr}$, progressive trends only started to become noticeable around two hours prior to failure. From this it can be concluded that analysis with larger time windows may be useful for early detection of the onset of slope failures.

\section{References}

Cabrejo-Liévano, AG 2013, 'Analysis of failures in open pit mines and consideration of the uncertainty when predicting collapses', in PM Dight (ed.), Proceedings of the International Symposium on Slope Stability in Open Pit Mining and Civil Engineering, Australian Centre for Geomechanics, Perth, pp. 483-498.

GroundProbe 2013, SSR-Viewer 8, software, version 8.1, www.groundprobe.com 